\title{
Psychoanalytische Praxis in der stationären psychiatrisch psychotherapeutischen Grundversorgung
}

Natalija Gavrilovic Haustein (Winterthur)

Zusammenfassung: Stationäre Behandlungen in psychiatrischen Kliniken sind zunehmend strukturellen Veränderungen ausgesetzt, die den Fokus vermehrt aufWirtschaftlichkeit legen. Psychoanalytische Haltungen, Theorien und Techniken sind wichtig und wirken. Trotz Zeit- und Kostendruck können sie auch in einer psychiatrisch-psychotherapeutischen Klinik der Grundversorgung eingesetzt werden. Ihre Anwendung wird hier für den Aufbau und den Betrieb einer Psychotherapiestation für Depressions- und Angsterkrankungen beschrieben. Neben dem psychotherapeutischen Einsatz, birgt die psychoanalytische Grundhaltung auch Chancen für die psychiatrische Notfallversorgung auf Akutstationen und die stationäre Krisenintervention. Dies wird anhand von praktischen Anwendungsbeispielen und der Frage nach Chancen in der psychoanalytischen Ausbildung aufgezeigt.

Schlüsselwörter: Psychoanalyse, Psychiatrie, stationäre Psychotherapie, Krisenintervention, Akutpsychiatrie

In den letzten Jahren ist ein strukturellerWandel in der Psychiatrielandschaft der Schweiz zu beobachten. Die allgemeine stationäre Aufenthaltsdauer wird immer kürzer, was vor allem die Konzepte auf Psychotherapiestationen beeinflusst.

Laut H+ (2015) lässt sich in einer Übersicht der wichtigsten Kennzahlen der Schweizer Spitäler und Kliniken in den Jahren 2001-2015 eine Entwicklung hin zu 23\% weniger Betten, 55\% mehr Hospitalisationen und $13 \%$ kürzere Aufenthaltsdauern in den Psychiatrien aufzeigen. Waren es im Jahr 2001 noch 37.3 Tage, verblieben die Patienten 2015 nur noch durchschnittlich 33.1 Tage in der psychiatrischen und psychotherapeutischen Klinik. Das heisst, es gab insgesamt weniger Betten, jedoch mehr und kürzere Hospitalisationen.

Eine weitere wichtige Entwicklung war die Einführung des TARPSY im Januar 2018. Beim TARPSY handelt es sich um ein Tarifsystem, welches alle gesamtschweizerischen stationären Leistungsbereiche der Erwachsenen-, Kinder- und 
Jugendpsychiatrie abdeckt. Es wird durch die Kern- und Arbeitsgruppen der SwissDRG AG (2016) erarbeitet und entspricht den Abrechnungsvorgaben des Krankenversicherungsgesetzes. Das Ziel ist, klare Rahmenbedingungen zu schaffen, um die Transparenz und Vergleichbarkeit von Kosten und Qualität zu erhöhen und dadurch den patientenorientierten Ressourceneinsatz zu fördern.

Es gibt dabei sogenannte leistungsbezogene Tagespauschalen. Dabei ist die Höhe der Pauschale abhängig von der Dauer des stationären Aufenthaltes und vom Schweregrad der Symptomatik. Sie sinkt mit zunehmender Aufenthaltsdauer. Somit wird ein finanzieller Anreiz geschaffen und die Wirtschaftlichkeit in den Vordergrund gestellt.

Diese strukturellen Veränderungen haben auch Einfluss auf den Inhalt der klinischen Tätigkeit. Sie drohen unsere Arbeit im stationären Alltag einzuengen, den Fokus zu verschieben und auf kurzfristigere stationäre Interventionen hinzuzielen. Die Behandlungen sollen schneller greifen und besser evaluierbar sein.

Die Psychoanalyse und ihre Anwendungsformen der psychoanalytischen Psychotherapie repräsentieren eine Form der Behandlung, welche auf ein vertieftes Verständnis innerer Vorgänge mit ihren unbewussten Anteilen zielt und nicht auf kurzfristige schnelle und oberflächliche Interventionen ausgerichtet ist. Das Subjekt wird als Ganzes betrachtet - mit all seinen Facetten: Lebensgeschichte, Beziehungsmuster, Erfahrungen sowie die Dynamik im Hier und Jetzt. Dies impliziert eine gewisse Behandlungsdauer, was den veränderten Strukturen in der Psychiatrielandschaft gerade in Hinblick auf die Dauer zu widersprechen scheint.

Haben die Psychoanalyse und ihre Anwendungsformen durch die veränderten Strukturen in der stationären psychiatrisch-psychotherapeutischen Grundversorgung überhaupt noch eine Daseinsberechtigung? Wie können psychoanalytische Konzepte nutzbringend eingesetzt werden? Warum sollten sie in einer psychiatrischen Klinik eine Rolle spielen?

\section{Eine Psychotherapiestation mit dem Fokus auf Depressions- und Angsterkrankungen}

Ich möchte am Beispiel einer Psychotherapiestation in einer psychiatrisch-psychotherapeutischen Klinik der Grundversorgung darstellen, wie psychoanalytische Methoden und Haltungen angewendet und nutzbringend in den Klinikalltag integriert werden können. Die Psychotherapiestation hat einen Fokus auf Depressions- und Angststörungen (DAS).

Bei den meisten Patientinnen, die auf der Depressions- und Angststation (DAS) behandelt werden, stehen affektive Erkrankungen wie Depressionen 
oder Angststörungen im Vordergrund. Dabei handelt es sich meist nicht um die «klassische» Depression auf neurotischem Niveau, sondern um rezidivierende Depressionen, denen eine deutliche strukturelle Vulnerabilität oder Beeinträchtigungen im Sinne von Persönlichkeitsakzentuierungen bis hin zu Persönlichkeitsstörungen zugrunde liegen. Rudolf (2007, S. 358) beschreibt, dass bei stationären Patienten insgesamt ein eher mässig bis gering integriertes Strukturniveau vorherrscht, so dass Patientinnen weitgehend durch strukturelle Beeinträchtigungen gekennzeichnet sind. Erfahrungsgemäss sind es eher strukturell schwächere Personen, die sich zur Behandlung auf eine Psychotherapiestation einer Klinik der psychiatrisch-psychotherapeutischen Grundversorgung begeben. Strukturstärkere Personen, die keinen stationären Aufenthalt benötigen, suchen eher störungsspezifische ambulante Angebote auf, wie z. B. Depressions- und Angstzentren. Besteht die Notwendigkeit einer stationären Behandlung, begeben sich solche Patienten eher in eine rein psychotherapeutische oder psychosomatische Klinik ohne die zusätzliche Akutpsychiatrie mit dem Auftrag der notfallmässigen Grundversorgung.

Ein weiterer Grund für vermehrt strukturschwache Patienten auf Psychotherapiestationen in Kliniken der Grundversorgung ist die Nähe zur Akutpsychiatrie und zum Kriseninterventionszentrum. Patientinnen, die dort notfallpsychiatrisch behandelt werden und sich aufgrund mangelnder Erfahrungen oder fehlender Auseinandersetzung nicht aus eigener Initiative in die stationäre psychotherapeutische Behandlung begeben würden, können so überhaupt erst den Zugang zur stationären Psychotherapie gewinnen. Dank infrastruktureller Versorgungsgegebenheiten durch kurze Wege zwischen den Akutstationen und den psychotherapeutischen Angeboten können so auch längere Wartezeiten umgangen werden.

Auf der DAS werden insgesamt 16 Patienten mit einer Aufenthaltsdauer von sechs bis acht Wochen behandelt. Die durchschnittliche Aufenthaltsdauer liegt bei 48 Tagen.

Das zugrundeliegende Behandlungskonzept orientiert sich an dem integrativen Modell der stationären Psychotherapie (Janssen, 1987). Bei diesem Modell wird das Übertragungsgeschehen auf verschiedene Ebenen wie Patientengruppe, Einzel- und Gruppentherapeutinnen, Pflegeteam, Spezialtherapeutinnen sowie auf die Klinik verteilt. Das gesamte interprofessionelle Team nimmt die Übertragung auf, welche nicht nur einer Person, sondern einem ganzen Team gilt und oftmals wie «aufgesplittert» erscheint. Das Team bearbeitet diese Übertragungsprozesse, versucht sie zu verstehen und mit der psychischen Welt der Patientin in Zusam- 
menhang zu bringen. Die Erkenntnisse werden dann in kleinen Portionen wie «vorverdaut» an die Patientin zurückgegeben, ohne dass eine emotionale Überforderung daraus resultiert. Im besten Fall kann die Patientin damit ihrem Seelenleben und Interaktionsverhalten etwas näherkommen.

Die Patientengruppe mit ihren vielfältigen zwischenmenschlichen Interaktionen und Beziehungserfahrungen stellt den wichtigsten Angelpunkt dar. Der Realitätsraum ist kaum vom Therapieraum getrennt, beide gehen ineinander über und vermischen sich. Somit wird jegliches Geschehen auf der Station zu psychotherapeutisch relevantem Material. Das Verbalisieren der Erlebnisse im stationären Alltag steht dabei im Vordergrund. Das eigene biographische Material dient zum besseren Verständnis der aktuellen Vorkommnisse in dieser Konstellation.

Die Behandlungsziele auf der DAS sind die Entwicklung eines individuellen Problembewusstseins, die Entdeckung eigener Fähigkeiten und das Erleben neuer Beziehungserfahrungen. Im Vordergrund steht vor allem das Übertragungs- und Gegenübertragungsgeschehen, ohne dass zu sehr mit der direkten Deutung gearbeitet wird. Als psychoanalytisch-psychotherapeutisches Grundmodell wurde hier die strukturstärkende Psychotherapie nach Gerd Rudolf (2013) vorgelebt und in den Stationsalltag eingebracht. Ich entschied mich dafür, den Schwerpunkt auf die strukturstärkende Therapie zu setzen, da sie mir passend für die strukturschwachen Patienten zu sein scheint und auch in der praktischen Umsetzung in der Behandlung und in der Weiterbildung verständlich und einfach umsetzbar ist. Im klinischen Alltag habe ich diese Therapieform als sehr hilfreich erlebt: Bei der Beschäftigung mit dem Strukturniveau geht es um ein schnelles Erfassen der Selbst- und Ich-Organisationen, insbesondere der Selbststeuerungs- und Interaktionsfähigkeit, damit die Patienten nicht überschätzt und deshalb ungeeignete Behandlungsstrategien angewendet werden. So kann ein Überblick über defizitäre und gelingende Strukturaspekte zuerst im Team und dann gemeinsam mit den Patienten erarbeitet werden (Rudolf, 2007, S. 357).

Es ist bekannt, dass gerade für eine strukturelle Verbesserung im Sinne einer Nachreifung, ein längerer therapeutischer Prozess notwendig ist. Gerade die klassisch ambulanten psychoanalytischen Therapien bieten mit ihren längerfristigen Behandlungsdauern diesen Rahmen. Die stationären psychotherapeutischen Behandlungen sind in Bezug auf ihre notwendige Dauer bisher kaum ausreichend untersucht (Klug \& Huber, 2019, S. 30). Erfahrungsgemäss gilt eine Dauer von 2-3 Monaten als angemessen und notwendig. Seit der Einführung des TARPSY im Jahr 2018 hat sich die allgemeine stationäre Behandlungsdauer deutlich reduziert. 
Diesbezüglich musste eine Alternative zu einer einzelnen längeren stationären Behandlung gefunden werden.

Aufgrund der relativ kurzen Aufenthaltsdauer von durchschnittlich 48 Tagen hat sich das Prinzip der «Intervalltherapie» eingebürgert. So müssen die Aufenthalte nicht weiter verlängert, sondern es kann die Möglichkeit eines erneuten Eintrittes mit einem bestimmten Behandlungsfokus nach einer «Belastungserprobung» im häuslichen Rahmen oder nach einer längeren Phase im Alltag angeboten werden. Der Druck diesbezüglich war sehr hoch, da seitens der Klinikleitung längere Aufenthalte (>45 Tagen) nicht mehr geduldet wurden. Klug und Huber (2019, S. 28) beschreiben sogar, dass mit der Intervalltherapie im stationären Rahmen bessere Erfolge erzielt werden als mit der zeitlichen Verlängerung einer Behandlung. Im klinischen Alltag hat sich die Intervalltherapie insofern bewährt, als dass damit zusätzlich den starken Regressionstendenzen bei strukturell eher schwachen Patienten im stationären Rahmen entgegengewirkt werden kann. Gleichzeitig stellt es eine Herausforderung für das gesamte Behandlungsteam dar, alle grundlegenden Elemente einer stationären psychotherapeutischen Behandlung wie Kennenlernen, Diagnostik, Beziehungsaufbau, Behandlungsphase und Therapiebeendigung bei so kurzer Aufenthaltsdauer unterzubringen. Eine enge Zusammenarbeit und ein stetiger Austausch sind dabei unabdingbar, aber auch anspruchsvoll, besonders bei personellen Engpässen. Auch in solchen Zeiten wird die psychoanalytisch orientierte Fallsupervision als äusserst hilfreich erlebt.

Für die Patientinnen wiederum stellt die Möglichkeit einer Intervalltherapie eine grosse Entlastung dar, da diese zu Beginn der Therapie oft ein gesteigertes Bedürfnis nach zwischenmenschlicher Zuwendung zeigen.

So hatten wir es mit schwer erkrankten Patientinnen zu tun, die auch noch in kürzerer Zeit behandelt werden sollten. Dies rief nach weiteren Anpassungen und kreativen Lösungen. Eine Möglichkeit war, die Arbeit mit dem Fokus auf wenige wichtige Behandlungsziele zu begrenzen. Aber auch eine schnellere und effizientere Diagnostik musste eingeführt werden, ohne dass es zu therapeutisch qualitativen Einbussen kam. Um inhaltlich-diagnostisch rasch und effizient einen Überblick zu erlangen, stellt sich die Anwendung der Operationalisierten Psychodynamischen Diagnostik OPD (Arbeitskreis OPD, 2009) als hilfreich dar. Bei der OPD handelt es sich um ein multiaxiales Diagnosesystem in Interviewform zur Ergänzung der Symptomdiagnostik aus psychodynamischer Perspektive. Gleichzeitig lassen sich damit auch Behandlungsfoki formulieren und festlegen. Auch wenn die OPDDiagnostik zu Beginn aufwendig erscheint, bringt sie im Verlauf eine deutliche Entlastung und Bereicherung. Bei der praktischen Umsetzung kommt es darauf 
an, wie ausführlich die OPD im klinischen Alltag genutzt wird. Die Erfahrungen zeigten, dass es sinnvoller war definierte Eingrenzungen zu machen. Die OPD sollte im klinischen Alltag «lebbar» und «anwendbar» sein, auch für Therapeutinnen die nicht über einen psychoanalytischen Hintergrund verfügen oder noch am Anfang ihrer Ausbildung stehen und für weitere Berufsgruppen wie Pflege, Spezialtherapeutinnen und Sozialdienst.

Neben der effizienteren Diagnostik struktureller Themen und der Festlegung definierter Behandlungsschwerpunkte konnten durch die Anwendung der OPD gerade interpersonelle Themen der Patientinnen über die sog. «Beziehungsachse» besser verstanden werden. Diese stellt die zweite psychodynamische Achse in der OPD dar und befasst sich mit der Diagnostik dysfunktionaler Beziehungsmuster. Sie bezieht sich sowohl auf das von den Patienten selbst wahrgenommene problematische Beziehungsverhalten als auch auf jene Beziehungsaspekte, die andere (d.h. auch der Untersucher) in der Begegnung mit dem Patienten erleben (Arbeitskreis OPD, 2009, S. 82). In der Therapie auftretende Übertragungsangebote von Patientinnen geben Raum für Reinszenierungen biografisch bedeutsamer Beziehungsfiguren und werden dadurch zum Material und Gegenstand der therapeutischen Arbeit. Solche Szenen der Übertragung werden in der Therapie aufgriffen und aufgezeigt.

Da das gesamte Team im Rahmen einer zweitägigen, durch die Klinik finanzierten Fortbildung geschult wurde, förderte dies auch eine gemeinsame psychodynamische Sprache sowie den Zusammenhalt der Station.

Die Anwendung aller fünf Achsen der OPD im klinischen Alltag ist wegen der strukturellen Gegebenheiten des Institutionsalltages zu aufwendig und daher nicht möglich. In der klinischen Umsetzung werden die Achsen I (Krankheitserleben und Behandlungsvoraussetzungen) und V (Psychische und psychosomatische Störungen nach ICD-10) vor allem im Rahmen von Vorgesprächen angewendet, um die vorläufigen Diagnosen zu benennen und eine Prognose stellen zu können. In Vorgesprächen werden neben einer ersten Einschätzung auch Behandlungsschwerpunkte gemeinsam mit der Patientin festgelegt. Während der stationären Behandlung wird in den zweimal wöchentlich stattfindenden Fallbesprechungen die Achse II (Beziehung) durch das interprofessionelle Kernteam, welches aus Pflege-Bezugsperson und ärztlicher oder psychologischer Therapeutin besteht, vorgestellt. Schilderungen von konkreten, schwierigen und konflikthaften Situationen mit anderen Menschen helfen dabei, Beziehungsmuster aufzudecken, unter Einbezug eigener Erwartungen und Befürchtungen sowie Reaktionen anderer. Im klinischen Alltag ist diese Achse am hilfreichsten, um 
schwierige zwischenmenschliche Situationen, auch im Kontakt zwischen Patienten und Pflegeteam, aufzudecken und besser zu verstehen. Die Achse III (Konflikt) und die Achse IV (Struktur) werden noch durch die fallführende Therapeutin oder die Oberärztin ergänzt. Dadurch wird nicht nur ein psychodynamisches Verständnis erarbeitet, sondern auch die Grundlage der Behandlung geschaffen. Übergeordnete Behandlungsziele werden abgeleitet, welche dann im wöchentlich durchgeführten interprofessionellen Rapport überprüft werden. Die OPD ist ein sehr nutzbringendes Beispiel derWeiterentwicklung der Psychoanalyse und deren Anwendungsmöglichkeit, um diese unter veränderten strukturellen Bedingungen auch im stationären Rahmen anzuwenden.

Weitere wichtige Pfeiler der stationären Therapie auf der Depressions- und Angststation sind die zweimal wöchentlich stattfindenden Einzelgespräche, in denen die erlebten Beziehungserfahrungen evaluiert, reflektiert und bestenfalls verinnerlicht werden können. Dabei gibt es keine strikte inhaltliche Trennung zwischen Einzel- und Gruppentherapie, Themen der Gruppentherapie können auch im Einzelsetting besprochen werden. So kann es vorkommen, dass die Einzeltherapeutin der Patientin in der Gruppentherapie begegnet. Die Gruppentherapie findet ebenfalls zweimal in der Woche statt und bedient sich psychodynamischer Elemente. Dabei sind die häufigsten Gruppenthemen die im Stationsalltag und an den Wochenenden erlebten Interaktionen. So werden vor allem die Schwierigkeiten eines Einzelnen mit seiner Umwelt reflektiert und verbundene Affekte deutlich gemacht. Die Empathie- und Mentalisierungsfähigkeit des Einzelnen wird dadurch gefördert. Die Therapeutinnen sind präsenter, stützender und strukturierender als in einer klassischen psychoanalytischen Gruppentherapie. Die einzelnen Gruppenmitglieder stehen im Vordergrund, weniger die Gruppe als Ganzes. Deutungen unbewussten Materials werden in Hinblick auf die eher strukturschwachen Patientinnen weniger angewendet.

Die milieutherapeutischen Angebote - morgendliche Rapporte, wie aber auch die Stationsversammlung - bilden den Rahmen, in dem die spezifischen Themen des engen Zusammenlebens thematisiert werden können. Ergänzungen stellen die Spezialtherapien im Einzel- und Gruppensetting dar, hier wird Kunst-, Ergo-, Bewegungs- und Physiotherapie angeboten. Diese werden nach Indikation auf die übergeordneten Ziele angewendet und sollen die Symbolisierungsfähigkeit der Patienten stärken. Für den gemeinsamen Austausch ist es von grosser Wichtigkeit, dass bei den Fallbesprechungen alle am Behandlungsprozess beteiligten Therapeutinnen anwesend sind. So können die jeweils abgespaltenen Erfahrungen 
der einzelnen Patientinnen vom Team zusammengeführt und besser verstanden werden.

Wenn es das Krankheitsbild erfordert, finden ergänzende verhaltenstherapeutische Interventionen statt, so zum Beispiel bei einer ausgeprägten Angstsymptomatik, die eine schnelle Entlastung notwendig macht.

Die Herausforderung im Alltag sind die hohen Anforderungen an die Reflexionsfähigkeit des Teams und die Vermeidung narzisstischer Überhöhungen in der Behandlung. Dafür bietet sich eine regelmässige psychoanalytisch orientierte Supervision an. Diese wird auf der DAS in monatlichen Abständen durchgeführt und hat einen primär fallorientierten Fokus, mit dem Ziel, die Dynamiken der Patientengruppe im Team zu reflektieren.

\section{Psychoanalyse in Akutsituationen}

Der nutzbringende Einsatz von psychoanalytisch orientierten Therapieansätzen auf einer Psychotherapiestation ist nachvollziehbar. Psychotherapeutische Elemente und eine psychoanalytische Haltung sollen auch in der Akutpsychiatrie ihren Platz finden. So kann eine qualitativ hochwertige und reflektierte Behandlung auf allen Ebenen gewährleistet werden.

Auf Akutstationen werden Patienten in schweren Krisen sowie auch akute psychiatrische Notfälle behandelt. Ein beachtlicher Teil der Patienten kommt gegen den eigenen Willen per Fürsorgerische Unterbringung (FU) als Zwangsmassnahme in die Klinik. Innerhalb der Behandlung ist es notwendig, sich rasch einen Überblick über die Thematik der Patientin zu verschaffen, sie einzuschätzen und als Gegenüber in diesem komplexen Gefüge handlungsfähig zu bleiben (Ehrenthal, 2017). Dies gilt besonders für die notfallmässige Behandlung auf der Akutstation. Patienten kommen meist mit Vorinformationen in Form eines Zuweisungsschreibens auf die Station. Noch wichtiger sind die verbalen und nonverbalen Auskünfte. Argelander (1970) und Lorenzer (1970) beschreiben das «szenische Verstehen» als eine Besonderheit des psychoanalytischen Settings. Auch auf der Akutstation als einem Sondersetting therapeutischer Behandlungen können wichtige Informationen im Sinne des «szenischen Verstehens» interpretiert werden. Es wird verstanden als die Wiederbelebung von «Interaktionsrepräsentanzen» in aktuellen therapeutischen Beziehungen (Happach, 2016, S. 114). Gerade beim Eintritt auf eine Akutstation sind diese Interaktionsrepräsentanzen deutlich zu spüren. Sie können als wichtige unbewusste und bewusste Mitteilungen des einzelnen Individuums dienen oder dessen Interaktionsmuster in Beziehungen darstellen. 
Dabei begleiten uns die grundlegenden psychoanalytischen Konzepte der Übertragung und Gegenübertragung. Auch wenn Akutstationen für den Übertragungsprozess nicht typisch sind, findet Übertragungsgeschehen auch hier statt. Die wiederholenden Inszenierungen zeigen sich stärker auf einer konkreten Handlungsebene als im Sprechen und Fantasieren, wofür das Setting eine Bühne bereitstellt (Gumz \& Storck, 2018, S. 46). Es ist wichtig solche zu erkennen, zu verstehen und nach Möglichkeit zu nutzen. Auch auf einer Akutstation gibt es geordnete und ruhige Momente, die es ermöglichen Übertragungsphänomene zu reflektieren. Davon ausgehend, dass das Gegenüber in der aktuellen neuen Beziehungssituation alte Erfahrungen miteinfliessen lässt und diese direkt auf die aktuelle Situation projiziert, können hier wertvolle Informationen zur Patientin ermittelt werden. Inwieweit mit dem Phänomen der Übertragung direkt gearbeitet werden kann, ist von Fall zu Fall unterschiedlich und sicherlich von der Behandlungsdauer und Schwere der Erkrankung abhängig.

Das Gleiche gilt für die Gegenübertragung. Sie bietet ebenfalls eine reichhaltige Quelle zum Verständnis der therapeutischen Situation. So wird der Therapeut selbst gleichsam zum Resonanzkörper dessen, was gerade passiert (Ehrenthal, 2017, S. 267).

Durch die Würdigung der Übertragung und Gegenübertragung sowie des szenischen Verstehens können im gesamten Behandlungsteam Hürden und Ängste abgebaut werden, was zu einer empathischeren und dadurch auch entlasteten Arbeitsbeziehung mit den Patienten beitragen kann. Eine regelmässig durchgeführte psychoanalytisch orientierte Fallbesprechungen, auch auf den Akutstationen, verhilft zu einem besseren Verständnis der Psychodynamik.

Das problematische und durchaus wichtige Thema «Zwang in der Akutpsychiatrie» darf an dieser Stelle nicht unbeachtet bleiben. Die psychiatrischen Kliniken haben sich im Laufe der Zeit auch diesbezüglich weiterentwickelt. Wissenschaftliche Untersuchungen werden durchgeführt und alternativ auch psychotherapeutische Methoden angewendet, um Massnahmen gegen den Willen der Patienten zu vermeiden oder sie zumindest so gering wie möglich zu halten. Gerade die «Doppelaufgabe» der Psychiatrie erschwert dies massgeblich. Auf der einen Seite sollen medizinische Behandlungen mit dem Ziel der Besserung der Gesundheit angeboten werden, auf der anderen Seite gibt es die gesellschaftlich auferlegte Aufgabe, dass die Psychiatrie sich bestimmter Personen annehmen soll, um diese temporär von der Gesellschaft zu separieren. Dazu gehören Situationen mit erhöhtem Gewaltpotential oder anderen Auffälligkeiten im Alltag, die nicht in erster Linie einen psychiatrischen Hintergrund haben müssen. Auch diese psych- 
iatrisierten Situationen führen dazu, dass in bestimmten Fällen von Selbst- und Fremdgefährdung weiterhin Zwangsmassnahmen angewendet werden. Eine grundsätzliche psychoanalytische Haltung fördert differenzierte Auseinandersetzungen auch mit dieser gesellschaftlich auferlegten Aufgabe.

Heute ist es selbstverständlich, dass Nachbesprechungen erfolgter Zwangsmassnahmen mit den Patienten durchgeführt werden. Diese Art der Reflektion ist eine Möglichkeit für Patienten und Mitarbeiter, Grenzüberschreitungen besser zu verstehen, zu bearbeiten und einbetten zu können. So wird die Gefahr von weiteren Traumatisierungen gemindert. Systematisch durchgeführte Nachbesprechungen können vorbeugend bei weiteren Zwangsmassnahmen in Notfall- und Krisensituationen sein. In diese Richtung zielt auch das Konzept der Behandlungsverträge. Hierbei handelt sich nicht um ein psychoanalytisches Konzept, es definiert jedoch die gemeinsame therapeutische Beziehung. Nach meiner Einschätzung fördern die systematischen Nachbesprechungen der Zwangsmassnahmen sowie die Behandlungsverträge die Beziehungs-, Mentalisierungs- und gegenseitige Empathiefähigkeit, gerade wenn es sich um Patienten mit wiederholten Aufenthalten in der Akutpsychiatrie handelt. Durch eine vertiefte persönliche therapeutische Beziehung können Vorbehalte und Vorurteile auf beiden Seiten abgebaut und bei einem erneuten Kontakt die «Heftigkeit» der Affekte beidseitig gemildert werden.

Happach (2016, S. 118 ff.) erwähnt in seiner Auseinandersetzung mit dem Thema der Beziehungsdynamik und der psychiatrischen Zwangsbehandlung einen weiteren interessanten Aspekt. Er bezieht sich auf das Mentalisierungskonzept als eine neuere Entwicklung des psychodynamischen Verständnisses, welches sich aus der Bindungstheorie entwickelt hat. Beim Eintritt auf die Akutstation in einem hilfsbedürftigen Zustand können Mentalisierungseinbrüche erfolgen, da in solch einem anspruchsvollen Moment das Bindungssystem aktiviert wird und so die Repräsentanzen frühkindlicher Erfahrungen reaktualisiert werden. Eine Aktivierung des Bindungssystems wird durch eine gemischte Situation von Not und Angst ausgelöst. Happach beschreibt diese Situation als Versuch der Wiederherstellung einer inneren Kohärenz. Es ist eine psychoanalytische Tradition, $\mathrm{zu}$ verstehen versuchen, wie Patienten Interventionen erleben und wie dadurch Rollen- und Erlebensmuster sichtbar werden.

Auf Akutstationen werden, neben anderen Krankheitsbildern, häufig Menschen mit Psychosen und Schizophrenien behandelt. Die Kultivierung eines psychodynamisch inspirierten Klimas, gerade in der therapeutischen Behandlung von Psychosen ist nach Mentzos (2011) wichtig. Die von schwerkranken und psy- 
chotischen Patienten erzeugten Gegenübertragungsgefühle bei dem gesamten Behandlungsteam können mit einem psychodynamischen Grundverständnis am ehesten erkannt und daraus resultierende Interaktionen besser verstanden werden. Es gilt «die schwierige Mischung zwischen empathischem Einfühlen und respektierter Distanz, also Zuwendung ohne Intrusion herzustellen». Mentzos beschreibt dies sogar als «das Beste, was man dem (psychotischen, Anm. der Autorin) Patienten bieten kann» (2011, S. 232).

Aus diesem Grund sollte die Tradition der psychoanalytischen Auseinandersetzung auch im Akutbereich Platz finden, da sie hier zum Verständnis und zur Erleichterung beiträgt. Sicherlich ist dies nicht im gleichen Rahmen wie auf einer psychotherapeutischen Station möglich. Angepasst an die akute Situation, können trotzdem wertvolle Erkenntnisse generiert werden. So kann das Verständnis für den Patienten als Subjekt mit all seinen Facetten, Anteilen und Verhaltensweisen erhöht, und in schwierigen Situationen können angstauslösende Momente auf Grund von Unwissenheit vermindert werden. Gleichzeitig hilft eine psychoanalytische Grundhaltung, eigene Emotionen in der Gegenübertragung besser zu erkennen und zu verstehen. Dies dient auch der Vermeidung schwieriger interpersoneller Zusammenstösse.

Als Ergänzung zur biologischen Psychiatrie und zum sozialpsychiatrischen Modell ist eine psychodynamische Grundhaltung in der Akutpsychiatrie wertvoll.

\section{Stationäre Krisenintervention auf der «Couch»?}

In der stationären Krisenintervention befinden sich Menschen, die bisherige Bewältigungsstrategien aufgrund unerwarteter sowie belastender Ereignisse nicht mehr anwenden können. Es handelt sich dabei um kurze stationäre Behandlungen von durchschnittlich sieben bis neun Tagen. Der Unterschied zur Behandlung auf einer Akutstation besteht darin, dass die Patienten sich freiwillig zur stationären Krisenintervention melden. Sie befinden sich nicht in einem derart schweren Ausnahmezustand und sind in der Regel stabiler als Patienten auf einer Akutstation.

Neben den allgemeinen Kriterien der Krisenintervention, welche auch in anderen Therapieschulen angewendet werden, gibt es klare psychoanalytische Konzepte, die dabei zum Tragen kommen. Hier finden wir erneut die Übertragung und Gegenübertragung als zentrale Konzepte psychodynamischer Theorie und Praxis, welche durch das Containment und das Arbeiten mit einem psychoanalytischen Fokus begleitet werden (vgl. Till, 2009, S. 773). Der Rahmen der Krisenintervention und der stabilere Zustand der Patienten bieten Raum für ein Übertragungsgeschehen, das in der Behandlung intensiv genutzt werden kann. 
Dabei werden psychoanalytische Kurzzeittherapien wie die Fokaltherapie oder die Intensive psychodynamische Kurzzeittherapie (Levenson, 2011, S. 26f.) zum Einsatz gebracht.

Allgemein sind für das Verständnis der aktuellen Krisensituation und den Bezug auf die subjektiven und individuellen Momente von Verletzlichkeit und Widerstandsfähigkeit Biografie und Persönlichkeitsfaktoren der betroffenen Person bedeutsam (Schauenburg, 2004, S. 153). Diese werden durch psychoanalytische Methoden des Erstgespräches erfasst. Das «szenische Verstehen» (Argelander, 1970; Lorenzer 1970) als wichtiges psychoanalytisches Einstiegselement kommt hier konkreter zum Tragen als in einer Akutsituation.

Ohne die Werkzeuge der psychoanalytischen Vorgehensweise - der Verknüpfung von vergangenen mit in der Behandlung erlebten Beziehungserfahrungen und dem Umgang mit unbewussten Konflikten und Verdrängungen - besteht die Gefahr, dass eine Krisenintervention sich nur an der Oberfläche abspielt. Das eigentlich «Zugrundeliegende» bleibt unberührt, wichtige Probleme unbeachtet und einer weiteren Krise bleiben Tür und Tor geöffnet. Die kurze Behandlungsdauer und das Setting stellen nicht die Voraussetzungen für eine Behandlung im Liegen auf der Couch dar, aber eine psychoanalytische Herangehensweise ist auch in der Krisenintervention nicht wegzudenken.

\section{Chancen für die Psychoanalyse}

Die Psychoanalyse und ihre Anwendungsformen bieten im klinischen Alltag ein vertieftes Verständnis für die Lage der Patienten. Moser (2006, S. 399) beschreibt: «Auch wenn in Institutionen keine eigentlichen, klassischen, Psychoanalysen durchgeführt werden, (ist) die Psychoanalyse ein Garant für die Respektierung des Subjektes als Ganzes und damit für ein menschliches, der Würde der Kranken angemessenes Milieu und eine entsprechende Therapie». Auch ergänzt er, dass die Psychoanalyse durch ihre Modifikationen der psychoanalytischen Psychotherapie damit für mehr Indikationen zugänglich gemacht worden ist. Es ist eine gewisse Aufklärungsarbeit, welche die Psychoanalyse damit leistet, mit dem Fokus auf das «Begreifenwollen» des Gegenübers.Ein weiterer Zusatznutzen in Bezug auf die Organisationstruktur und innerhalb der Teams einer psychiatrischen Klinik ist das psychodynamische Grundverständnis, womit auch interne Probleme besser erkannt und Abläufe optimiert werden können.

In den letzten Jahren beobachte ich wieder einen vermehrten Zuwachs an jungen Ärztinnen und Psychologinnen, die sich für die Psychoanalyse interessieren. In der Klinik, in der ich tätig bin, haben wir eine grosse Gruppe von 
jungen Kolleginnen und Kollegen, die sich psychoanalytisch weiterbilden. Auf die Frage, was sie für die Psychoanalyse motiviert, höre ich ein vertieftes Interesse an Ursachen und Dynamiken sowie komplementären Betrachtungsweisen, weil ihnen sonst «etwas fehle». Vielleicht sind gerade wir Psychoanalytiker für «das, was fehlt» zuständig. Wir bieten diese vertiefte und kritische Auseinandersetzung mit psychologischen, kulturellen und psychotherapeutischen Themen.

Daher bin ich optimistisch, was die Zukunft der Psychoanalyse angeht. Dies impliziert jedoch, dass sich auch die Psychoanalyse der heutigen Zeit anpassen muss ohne ihre Wurzeln zu vergessen oder zu verleugnen. Auch weitere Therapieschulen, wie die der Verhaltenstherapie (VT) haben sich weiterentwickelt. Die dritte Welle der kognitiv-verhaltenstherapeutischen Therapien wurde mit Konzepten aus der Psychoanalyse ergänzt. Beispiele sind hier der Aspekt der Übertragung und Gegenübertragung im Cognitiven Behavioral Analysis System of Psychotherapy (CBASP) und in der Schematherapie. Diese Tatsache zeigt, dass sich die psychoanalytische Betrachtungsweise auch in diesem Umfeld als nutzbringend erweist.

Indem dem Nachwuchs diese Betrachtungsweisen mit ihren Anwendungsmöglichkeiten nähergebracht werden, können die psychoanalytische Psychotherapie und auch die Psychoanalyse weiterwachsen. Dies fordert jedoch, dass es genügend psychoanalytisch orientierte Kolleginnen und Kollegen gibt, die sich für die Arbeit in psychiatrischen Institutionen interessieren und nicht direkt in die private Praxis gehen. Die Identität der Analytikerin im Rahmen einer psychiatrischen Institution ist oft keine einfache, manchmal sogar eine zwiespältige, da sie auch von den niedergelassenen psychoanalytischen Kolleginnen kritisch in Frage gestellt wird.

Die Mittel der Psychoanalyse und der psychoanalytischen Psychotherapie können bei einer breiten Palette an Störungsbildern nutzbringend eingesetzt werden. Dies beweisen auch die Weiterentwicklungen psychoanalytischer Anwendungsmethoden und -möglichkeiten mit ihren unterschiedlichen störungsspezifischen Ansätzen wie der Behandlung von Persönlichkeitsstörungen, Psychosen, Depressionen und somatoformen Störungen, um nur einige zu nennen. Es existieren viele Manuale zu psychoanalytischen Behandlungsmöglichkeiten unterschiedlicher Erkrankungsbilder.

Eine gewisse Manualisierung war ein notwendiger Entwicklungsschritt der Psychoanalyse in Richtung ihrer Anwendbarkeit im heutigen klinischen Alltag. Dies belegt, dass die Psychoanalyse es verstanden hat, sich den heutigen Gegebenheiten anzupassen, um weiterhin anwendbar zu bleiben. 
Die grosse Herausforderung hierbei ist, dass sie sich weiterhin ihrerWurzeln bewusst bleibt, damit die psychoanalytischen Prinzipien und Erkenntnisse in ihrer ursprünglichen Form ebenfalls erhalten bleiben und weitergegeben werden können.

\section{Literatur}

Arbeitskreis OPD (2009). OPD-2. Operationalisierte Psychodynamische DiagnostikDas Manual für Therapieplanung und Diagnostik. Göttingen: Hogrefe.

Argelander, H. (1970). Das Erstinterview in der Psychotherapie. Darmstadt: Wissenschaftliche Buchgesellschaft.

Ehrenthal, J. (2017). Psychodynamische Psychotherapie-Grundlagen, Wirksamkeit, Methoden, Techniken. PSYCH up2date 2017, 11, 267-286.

Gumz, A. \& Storck T. (2018). Übertragung und Gegenübertragung. In A. Gumz \& S. Hörz-Sagstetter (Hrsg.), Psychodynamische Psychotherapie in der Praxis (S. 43-56). Weinheim: Beltz.

Happach, C. (2016). Patientenautonomie, Psychodynamikund Psychopharmakologie: Die Beziehungsdynamik der psychiatrischen Zwangsbehandlung-zwischen medizinisch indizierter Behandlungsnotwendigkeit und Enactment. In J. Küchenhoff (Hrsg.), Psychoanalyse und Psychopharmakologie Grundlagen, Klinik, Forschung (S. 109-128). Stuttgart: Kohlhammer.

H+ Die Spitäler der Schweiz (2015). Spital- und Klinik-Monitor. Leistungen, Strukturen, Entwicklungen und Trends der Branche. Psychiatrie. Bern: H+, https:// www.hplus.ch/de/zahlen-statistiken/h-spital-und-klinik-monitor/ psychiatrie.pdf [18.10.2019].

Janssen, P. (1987). Psychoanalytische Therapie in der Klinik. Stuttgart: Klett-Cotta. Klug, G. \& Huber, D. (2019). Psychodynamik kompakt: Wie viel Zeit braucht der Mensch, um sich in der psychodynamischen Therapie zu verändern? Göttingen: Vandenhoeck \& Ruprecht.

Levenson, H. (2011). Psychodynamische Kurzzeittherapie. München: Reinhardt.

Lorenzer, A. (1970). Sprachzerstörung und Rekonstruktion. Frankfurt a. M.: Suhrkamp.

Mentzos, S. (2011). Lehrbuch der Psychodynamik-Die Funktion der Dysfunktionalität psychischer Störungen. Göttingen: Vandenhoeck \& Ruprecht.

Moser, A. (2006).Zukünftige Entwicklungen aus psychoanalytischer Sicht. In H. Böker (Hrsg.), Psychoanalyse und Psychiatrie: Geschichte, Krankheitsmodelle und Therapiepraxis. Heidelberg: Springer. 
Rudolf, G. (2007). Strukturbezogene Psychotherapie. In G. Rudolf \& P. Henningsen (Hrsg.), Psychotherapeutische Medizin und Psychosomatik-Ein einführendes Lehrbuch auf psychodynamischer Grundlage (S. 357-363). Stuttgart: Thieme.

Rudolf, G. (2013) Strukturbezogene Psychotherapie - Leitfaden zur psychodynamischen Therapie struktureller Störungen. Stuttgart: Schattauer.

Schauenburg, H. (2004). Psychodynamische Aspekte der Krisenintervention. In A. Riecher-Rössler, P. Berger, A. Yilmaz \& R. Stieglitz (Hrsg.), Psychiatrischpsychotherapeutische Krisenintervention (S. 153-164). Göttingen: Hogrefe. SwissDRGAG (2016). Das Wichtigste zu TARPSY auf einen Blick Stand Mai 2016. Bern: SwissDRGAG, https://www.swissdrg.org/application/files/2114/8104/2944/ Die_wichtigsten_Ziele_von_TARPSY-d.pdf [18.10.2019]

Till, W. (2009). Psychoanalytische Aspekte in der Krisenintervention. Psyche Zeitschrift für Psychoanalyse und ihre Anwendungen 63 (8), 773-793.

\section{Angaben zur Autorin}

Natalija Gavrilovic Haustein, 1975. Fachärztin für Psychiatrie und Psychotherapie FMH, Psychosomatische und Psychosoziale Medizin SAPPM. Psychoanalytikerin PSZ und Vorstandsmitglied EFPP. Langjährige institutionelle Erfahrung, zuletzt als Oberärztin auf einer Psychotherapiestation für Depressions und Angsterkrankungen, aktuell leitende Ärztin Akutpsychiatrie Integrierte Psychiatrie Winterthur - Zürcher Unterland (ipw); zusätzlich private Praxistätigkeit in Winterthur.. 